\title{
Escuta qualificada como ferramenta de humanização do cuidado em saúde mental na Atenção Básica
}

\author{
Angélica Brandão Santos
}

\section{RESUMO}

Motivação: A hipótese norteadora da investigação foi a de que a escuta qualificada na atenção primária a saúde é uma ferramenta capaz de revolucionar a lógica biomédica de cuidado.

Método: Este artigo orientou-se pelos preceitos de uma pesquisa bibliográfica, a fim de, coletar, analisar e interpretar aquilo que já foi dito, escrito ou filmado sobre escuta qualificada, cuidados primários e humanização em saúde mental. Posterior ao levantamento bibliográfico a autora efetuou um estudo exploratório com o intuito de elucidar o fenômeno pesquisado através das contribuições teóricas já existentes.

Resultados: A partir do referido estudo, a hipótese norteadora, pode ser comprovada, e os objetivos específicos possibilitaram que o potencial da escuta qualificada enquanto ferramenta de humanização do cuidado fosse explanado no decorrer da pesquisa.

Conclusões: A escuta qualificada possibilita a humanização das práticas de promoção e prevenção, diagnóstico, tratamento e reabilitação em saúde mental, ou seja, possibilita que neste contexto que consideradas as circunstâncias sociais, éticas, educacionais e psíquicas dos sujeitos envolvidos.

Palavras-chave: Humanização, Saúde Mental, Atenção Básica. 


\section{INTRODUÇÃO}

Desde os primórdios da civilização o homem segrega aquilo com o que não consegue lidar, a exemplo do que aconteceu com a lepra e algumas outras doenças, conforme descreve Foucault ${ }^{1}$ (1978) no livro intitulado "A história da Loucura". Com relação à insanidade não seria diferente, encarcerar os alienados, como eram inicialmente chamadas às pessoas com transtornos mentais, talvez fosse uma forma de lidar com a impotência humana diante de tal.

No Brasil, antes do movimento da Reforma Psiquiátrica, tratar a doença mental era sinônimo unicamente de excluir e asilar. $\mathrm{Na}$ exclusão da pessoa com transtorno mental do convívio social o exercício de seus direitos e deveres enquanto cidadão e também sua capacidade de autonomia foram negligenciados. O hospital psiquiátrico perpetuava a violência institucional que, por sua vez, agravava o sofrimento decorrente do quadro psicopatológico, o que ia de encontro com o que prega hoje a Política Nacional de Humanização e as práticas que consideram a integralidade do sujeito, ambas viabilizadas pelo movimento de Reforma Sanitária, do qual se originou uma nova forma de fazer saúde, institucionalizada pela Constituição de 1988, e regulamentada pela Lei no 8.080/90.

A partir de então, a Reforma psiquiátrica tomou fôlego e começou-se a pensar em novas alternativas e equipamentos que pudessem possibilitar tratamento digno as pessoas com transtorno mental. Com a promulgação da Lei $n$ 으 $10.216 / 2001$, os direitos das pessoas com transtorno mental foram instituídos e o modelo assistencial reordenado, sendo preconizado o alcance da recuperação através da inserção na família, trabalho e comunidade. A incorporação da Atenção Básica como dispositivo da Rede de Atenção Psicossocial (RAPS) possibilitou também a incorporação de práticas preventivas e promotoras da saúde mental.

Entretanto, em tempos de caos político nacional, em tempos de retrocesso no âmbito da saúde, em tempos em que a partir da Emenda Constitucional no 95 instituiu-se novo regime fiscal no que tange aos Orçamentos Fiscais e da Seguridade Social da União, que vigorará por vinte anos, congelando os investimentos em saúde e educação, em tempos em que o Hospital Psiquiátrico novamente foi incorporado a Rede de Atenção Psicossocial (RAPS) é de suma importância que produções científicas norteiem e sirvam de subsídios para despertar senso crítico quanto a práticas humanizadas de atenção à saúde mental, orientando assim acadêmicos, profissionais e a comunidade em geral.

Comparar os avanços após a Reforma Sanitária e a Reforma Psiquiátrica, bem como, as mudanças ocorridas no exercício dos cuidados ao portador de transtorno mental, na prevenção do adoecimento e promoção da saúde, e ainda, sublimar a relevância da escuta qualificada como método humanizador nesta esfera pode sensibilizar a sociedade geral e ser elemento protetor do progresso atingido, impedindo retrocessos. Portanto, estas se configuram a relevância social e acadêmica desta construção científica.

A hipótese que se buscou comprovar no transcurso desta produção foi de que a escuta qualificada constitui-se uma ferramenta capaz de revolucionar a lógica tradicional do cuidado em saúde mental no âmbito da Atenção Básica ( $A B)$. Para tanto, como escopo principal delineou-se o seguinte: Verificar o potencial da escuta qualificada como ferramenta de humanização do cuidado em saúde mental no âmbito da Atenção Básica em saúde no Brasil.

Assim, para que o objetivo elementar acima descrito pudesse ser atingido foram estabelecidos os seguintes objetivos específicos: Contrastar a forma de cuidado tradicional em saúde mental e o que preconiza a reforma psiquiátrica no Brasil; Explanar as competências da Atenção Básica no que tange aos cuidados em saúde mental; Identificar a relevância da escuta qualificada como ferramenta de Humanização em Saúde.

Este artigo orientou-se pelos preceitos de uma pesquisa bibliográfica, a fim de, coletar, analisar e interpretar aquilo que já foi dito, escrito ou filmado sobre escuta qualificada, cuidados primários e humanização em saúde mental. Posterior ao 


\section{APS em Revista}

Vol. 1, n. 2, p. 170/179 | Maio/Julho - 2019

ISSN 2596-3317 - DOI 10.14295/aps.v1i2.23

Santos, A. B.

levantamento bibliográfico a autora efetuou um estudo exploratório com o intuito de elucidar o fenômeno pesquisado através das contribuições teóricas já existentes.

A partir dos seguintes descritores: "Humanização em Saúde", "Humanização em Saúde Mental", "Atenção Básica e cuidados primários em Saúde Mental", "Legislação regulamentadora da Atenção em Saúde Mental", "Política Nacional da Atenção Básica", "Política Nacional de Humanização" foram consultados artigos, dissertações, teses e livros, indexados nos bancos de dados LILACS (Literatura Latino Americana e do Caribe em Ciências Sociais), Scielo (Scientific Eletronic Library OnLine) e Google Scholar (Google Acadêmico), assim como, obras físicas do acervo pessoal da autora.

O material utilizado para extração dos dados foi selecionado levando em consideração o objetivo principal e os objetivos específicos da pesquisa, é importante ressaltar que só foram verificados aqueles cujo título estivesse relacionado a estes. A análise das informações, por sua vez, também considerou os escopos previamente estabelecidos fornecendo assim, a partir da sua realização, subsídios para que os desígnios desta investigação científica fossem alcançados e a hipótese que a norteia fosse comprovada.

\section{A ESCUTA QUALIFICADA E SEU}

\section{POTENCIAL HUMANIZADOR DO}

\section{CUIDADO EM SAÚDE MENTAL NA}

\section{ATENÇÃO}

Conforme postulam Gonçalves e Sena² (2001), a ciência a despeito dos fenômenos sociais e a historicidade dos fatos é caminho para elucidar as contradições e as determinações presentes na história da humanidade. E quanto às mudanças ocorridas na realidade social, econômica, politica e cultural que impulsionaram a reforma psiquiátrica, as autoras supracitadas salientam que não ocorreram por acaso, mas que foi fruto de um movimento de revolução e transformação com repercussões na vida das pessoas com transtornos mentais, sua família e na sociedade. Desse modo, é primordial que se discorra sobre como transcorreu a evolução dos cuidados em saúde mental, o que se fará a seguir.

\section{Contextualização histórica do cuidado em Saúde Mental no Brasil}

No Brasil, a única resposta à doença e ao doente mental até meados da década de 90 era a internação, embora o movimento da Reforma Psiquiátrica tivesse iniciado em torno de 20 anos antes. Portanto, o modelo hospitalocêntrico /institucionalizante imperava, sem que houvessem equipamentos substitutivos, e romper com tal modelo tornou-se o maior bandeira da Luta Antimanicomial como também ficou conhecido o movimento a que se referiu anteriormente (FURTADO, CAMPOS 3 , 2005).

Tanto a Reforma Psiquiátrica, quanto a Reforma Sanitária, que acorreram no país concomitantemente, tinham por objetivo reformular os modelos de gestão e de assistência, de defesa da saúde coletiva, de equidade, bem como a participação de trabalhadores nos processos de gestão e tecnologia do cuidado. Desse modo, o primeiro, promoveu a ruptura da centralidade do modelo psiquiátrico segregador da população com transtornos mentais, que as demarcava como incapaz de compartilhar sua vida no social (FONSECA, JAEGER4, 2012). Como vertente principal do movimento supracitado se anunciou a desinstitucionalização com consequente desconstrução do manicômio, bem como, de seus paradigmas e sua substituição por outras práticas terapêuticas (GONÇALVES, SENA², 2001). Além disso, as pessoas com transtornos mentais passaram a ser enxergadas como sujeitos de direitos e atenção a elas dispensada já não tinha como objetivo apenas o controle da sintomatologia, mas a possibilidade de conferirIhes autonomia para o exercício da plena cidadania (BRASIL5, 2013). A partir da década de 90 , os movimentos sociais, inspirados no Projeto de Lei de Paulo Delgado, conquistam a aprovação em alguns estados brasileiros das primeiras leis que determinam a troca progressiva de leitos psiquiátricos por uma rede integrada de 
atendimento. Entretanto, aquele projeto de lei, só foi sancionado no Congresso Nacional em 2001, originando a Lei Federal no 10.216/2001 que dispõe sobre os direitos das pessoas com transtornos mentais e redireciona a assistência, privilegiando a oferta de tratamento de base comunitária (BRASIL6, 2001; BRASIL5, 2013). Em 2011, é promulgada a portaria 3.088 do Ministério da Saúde (MS) que institui a Rede de Atenção Psicossocial (RAPS) para pessoas com sofrimento ou transtorno mental e com necessidades decorrentes do uso de crack, álcool e outras drogas, no âmbito do Sistema Único de Saúde (SUS). Nos termos desta portaria a Atenção Básica $(A B)$ formada pelos seguintes pontos de atenção: a) Unidade Básica de Saúde (UBS); b) equipe de atenção básica para populações específicas: 1 . Equipe de Consultório na Rua; 2. Equipe de apoio aos serviços do componente Atenção Residencial de Caráter Transitório; c) Centros de Convivência passam a ser reconhecidos como dispositivos da RAPS (BRASIL7, 2011).

\section{Atenção Básica como dispositivo da}

\section{Rede de Atenção Psicossocial (RAPS)}

Segundo salienta Brasil8 (2017) na Política Nacional da Atenção Básica (PNAB), a Atenção Básica $(A B)$ se caracteriza por um conjunto de ações de saúde no âmbito individual, familiar e coletivo que envolve a promoção, prevenção, proteção, diagnóstico, tratamento, reabilitação, redução de danos, cuidados paliativos, bem como, vigilância em saúde, que devem ser desenvolvidas através de práticas de cuidado integrado e gestão, sob a responsabilidade de equipes multiprofissionais tendo como público alvo população em território definido, sobre as quais as equipes assumem responsabilidade sanitária. Dentro da Rede de Atenção Psicossocial são pontos de Atenção Básica os seguintes serviços, de acordo com Brasil7 (2011): 1) As Unidades Básicas de Saúde (UBSs), que se constituem como um serviço formado por equipes multiprofissionais cujo objetivo é desenvolver a atenção integral que impacte a situação de saúde e autonomia das pessoas e os determinantes e condicionantes da saúde coletiva. Assim, responsabilizam-se por um conjunto de ações no âmbito individual e coletivo que abrange a promoção e a proteção, a prevenção de agravos, o diagnóstico, o tratamento, a reabilitação, a redução de danos e a manutenção da saúde. 2) Equipes de Atenção Básica para populações específicas: a) Consultório na Rua, o qual pode ser definido com uma equipe multiprofissional que atua de forma itinerante e promove ações e cuidados de saúde para populações em situação de rua em geral, pessoas em situação de rua com transtornos mentais, e pessoas em situação de rua em decorrência do uso de crack, álcool e outas drogas. b) Equipe de apoio aos serviços do componente Atenção Residencial de Caráter Transitório, que por sua vez, oferecem suporte clínico e apoio a esses pontos de atenção. 3) Centro de Convivência, que é a unidade pública, articulada à Rede de Atenção à Saúde (RAS), em especial à RAPS, em que é ofertada a comunidade em geral espaços de sociabilidade, produção e intervenção na cultura e na cidade. Este dispositivo é estratégico no que tange a inclusão social de pessoas com transtornos mentais e pessoas que usam crack, álcool e outras drogas, já que, são espaços de convívio e sustentação de diferenças na comunidade. Ainda de acordo com o que postula BRASIL7 (2011), como responsabilidade da UBS dentro da RAPS pode-se elencar o desenvolvimento de ações de promoção e prevenção em saúde mental, cuidado dos transtornos mentais, assim como, ações de redução de danos e cuidado para usuários de crack, álcool e outras drogas. E no alcance desses objetivos, os profissionais que atuam nas UBSs podem contar com o apoio dos Núcleos Ampliados de Saúde da Família e Atenção Básica (NASF-AB), que são equipes multiprofissionais com responsabilidade de apoiar as equipes que a eles estiverem vinculadas, tanto no contexto técnicopedagógico como no contexto clínico-assistencial. É de suma importância ressaltar que a $A B$ se configura porta de entrada SUS, inclusive daquelas pessoas que demandam cuidados em Saúde Mental e que suas ações são desenvolvidas em um território geograficamente conhecido, o que possibilita aos profissionais da saúde proximidade e vínculo com os usuários e sua história de vida, 
portanto o cuidado é tático, já que facilita o acesso das equipes aos usuários e vice-versa (BRASIL5, 2013).

\section{Escuta qualificada e humanização do cuidado em Saúde Mental na Atenção Primária}

Antes de correlacionar a escuta qualificada a praticas humanizadas em Saúde Mental, convém fazer uma breve explanação sobre o conceito de humanização e o contexto histórico em que ela assumiu destaque dentro deste cenário. Pois bem, tal termo faz referência ao resgaste do respeito à vida humana, levando-se em consideração as circunstâncias sociais, éticas, educacionais e psíquicas implicadas em todo relacionamento humano (BRASIL9, 2001). Não é de hoje que práticas humanizadas de atenção e cuidado vêm sendo discutidas no contexto mundial. Como marco histórico neste campo Fortes10 (2004) aponta a Declaração da Conferência sobre os Cuidados em Saúde Primária, popularmente conhecida como declaração de Alma-Ata, uma vez que, esta reafirmou a saúde como um completo bem estar físico, mental e social, não se restringindo a ausência de doenças e que deve ser compreendida como um direito fundamental de todo cidadão. $O$ processo de humanização originou-se nos movimentos da Reforma Sanitária, nas Conferências de Saúde e nos grupos militantes da época como resposta a tensão, sofrimento e insatisfação enfrentados pelos profissionais e pacientes frente à violência institucional. Nacionalmente, este processo formalizou-se com a promulgação da constituição de 1988 e com a criação SUS, responsáveis por reordenar teoricamente, operacionalmente e romper com os velhos paradigmas do modelo biomédico de cuidado (Rios11, 2008).

Passadas uma década e meia da criação do SUS constatou-se que embora o sistema carregasse em suas entranhas princípios e diretrizes do que poderia ser a grande política de humanização nacional, inúmeras situações dentre as quais podemos citar as longas filas de espera, a falta de qualificação dos profissionais e falta de recursos, evidenciavam uma extensa distância entre a proposta humanizadora e a realidade do país. A partir do reconhecimento das falhas no sistema o Estado optou por implantar a Política Nacional da Humanização (PNH) com o objetivo de promover a valorização dos vários sujeitos nos processos de produção das práticas sanitárias (SIMÕES12, et al., 2007; BRASIL13, 2004).

A PNH apresenta como escopo principal a possibilidade de acolhimento apropriado e escuta qualificada dos seus atores, a saber: usuários, gestores e colaboradores. Assim, práticas de saúde não devem centrar-se na doença, mas sim no sujeito, uma vez que, este é sempre maior que seu diagnóstico estabelecido. A política tem por princípios a transversalidade, a indissocialidade entre atenção e gestão, bem como, o protagonismo, a corresponsabilidade e a autonomia dos sujeitos e coletivos. E, orienta-se pelas diretrizes descritas a seguir: acolhimento; gestão participativa e cogestão; ambiência; clínica ampliada e compartilhada; valorização do trabalhador; defesa do direito dos usuários. (RAIMUNDO, CADETE14, 2012; BRASIL15, 2013).

Nesta perspectiva, a escuta qualificada possibilita o encontro com a subjetividade do indivíduo, e pode ser definida como o ato de estar sensível ao que é comunicado e expresso através de gestos e palavras, ações e emoções (SANTOS16, 2014). Portanto, se desenha como uma importante ferramenta para que as diretrizes orientadoras da PNH possam, de fato, nortear a produção e gestão dos cuidados e dos processos de trabalho nos nível primário, secundário e terciário de atenção à saúde.

A qualificação do diálogo possibilita que o usuário seja atendido de forma integral, que o vínculo terapêutico seja estabelecido, que a diversidade e a singularidade prevaleçam no encontro entre quem cuida e quem recebe o cuidado, que haja possibilidade de equilíbrio entre os danos e benefícios gerados pelas práticas de sanitárias, e corresponsabilidades destas, reforçando o protagonismo do sujeito alvo de tais práticas (MAYNART17, et al., 2014; RAIMUNDO, CADETE14, 2012). 
A partir do momento que o profissional se disponibiliza a ouvir de forma ética e empática o usuário é possível acolher e criar vínculo. Sobre o vínculo e acolhimento Jorge17 et al. (2011, p. 3053) discorrem:

São decisivos na relação de cuidado entre o trabalhador de saúde mental e o usuário. Nesta relação, o acolhimento e o vínculo facilitam a construção da autonomia mediante responsabilização compartilhada e pactuada entre os sujeitos envolvidos nesta terapêutica.

Neste sentido, Brasil5 (2013) ressalta que por vezes o usuário não se dá conta de seus conflitos e sofrimentos com aquilo que fala, pensa ou faz, e que o profissional da Atenção Básica pode atuar como interlocutor viabilizando o manejo das angústias cotidianas que podem gerar somatizações ou complicações clínicas. O autor ainda argumenta que pelo fato da escuta ser uma prática comum no dia-a-dia das pessoas, mesmo daquelas sem formação no campo sanitário, atribui-se a ela um menor valor em detrimento de outras condutas técnicas, entretanto ela possibilita ao usuário e profissional a percepção e significação do sofrimento, além de elaboração de um plano terapêutico que atenda as especificidades do indivíduo.

Segundo Mendes19 et al. (2009) humanização envolve respeito pela integridade, dignidade e autonomia do paciente, e conforme Brasil5 (2013) evidencia, os cuidados em saúde mental devem possibilitar o exercício da plena cidadania do sujeito, por conseguinte, escutá-lo com qualidade seria o processo base para conhecer o sujeito, sua subjetividade, os determinantes do seu adoecimento, assim como, para elaborar um plano terapêutico que resgate o respeito a sua dignidade humana.

Há de se ressaltar ainda, que hoje, o cuidado em saúde mental não tem como alvo apenas o indivíduo já adoecido, através da Atenção Primária busca-se prevenir o surgimento dos transtornos mentais, a eliminação dos fatores de risco, e promoção da saúde em seu prisma biopsicossocial, ou seja, é primordial promover novas possibilidades de modificar e qualificar as condições e modos de vida. Neste ponto da RAS as intervenções devem ser construídas no cotidiano dos encontros de profissionais e usuário, devem ser criadas ferramentas e táticas que possibilitem suporte emocional aos pacientes (BRASIL5, 2013). O modelo hospitalocêntrico que se perpetuou como única forma de intervenção ao adoecimento mental já estabelecido tem na $A B$ uma alternativa de tratamento humanizado.

\section{CONSIDERAÇõES FINAIS}

Adiante seguem as considerações concernentes a cada escopo delineado para esta averiguação científica. Convém sobrelevar que a exploração do conhecimento pré-existente acerca da temática investigada foi possibilitada a partir da análise integrativa dos dados, realizada de forma sistemática levando em consideração os objetivos de antemão estabelecidos e a hipótese norteadora.

Pois bem, quanto ao primeiro intento específico, a saber: contrastar a forma de cuidado tradicional em saúde mental e o que preconiza a Reforma Psiquiátrica no Brasil, pode-se afirmar que por forma tradicional entende-se as práticas executadas no país antes da Reforma Psiquiátrica, orientada pelas premissas do modelo biomédico e hospitalocêntrico. Nestes moldes, o portador de transtorno mental era internado em hospitais psiquiátricos, as internações, por sua vez, eram longas, assim o paciente muitas vezes perdia o vínculo com familiares e com a comunidade a qual pertencia, não se pretendia devolver autonomia ao paciente e somente os aspectos saúde-doença eram considerados.

Durante o movimento, novas alternativas de tratamento passaram a ser cogitadas como substitutivas às anteriores, já que, estas estavam atreladas a mais sofrimento que o próprio adoecimento. Assim, com a promulgação da Lei $n$ 은 10.216/01, foram institucionalizados os direitos das pessoas com transtorno mental e passou-se a 
preconizar que o seu público alvo tivesse acesso a melhor terapêutica de saúde, tratamento humano e respeitoso, além de ser protegido contra qualquer forma de abuso e exploração. A partir de então, iniciou-se a elaboração de uma nova Rede de Atenção Psicossocial, na qual até meados do ano 2017 o hospital psiquiátrico não estava inserido.

A modelo de atendimento recomendado pela lei da Reforma Psiquiátrica é aquele que possibilite que o indivíduo não perca o vínculo com sua comunidade e família, que o tratamento seja realizado preferencialmente em serviços comunitários de saúde mental, com os meios menos invasíveis possíveis e que as internações necessárias sejam realizadas no hospital geral, apenas pelo tempo necessário, o que vale mais uma vez ressaltar, mudou com reincorporação do hospital psiquiátrico na RAPS.

Quanto às competências da Atenção Básica no que tange aos cuidados em saúde mental, que se configura $o$ segundo objetivo específico estabelecido, considera-se que é um nível estratégico para possibilitar o diagnóstico, tratamento e reabilitação próximos ao espaço ao qual o indivíduo pertence. Ademais, já que os profissionais possuem vínculo com o território e moradores devem conhecer a realidade de ambos, o que possibilita diagnoses e intervenções precoces e integrais. É também o nível de atenção encarregado de realizar práticas de prevenção, bem como, de promoção da saúde mental.

Já sobre o terceiro objeto específico proposto pela autora, que por sua vez, foi identificar a relevância da escuta qualificada como ferramenta de Humanização em Saúde, a partir da análise dos dados levantados, foi possível observar que a escuta atenta a tudo que é comunicado tanto pela linguagem verbal como pela não verbal, isenta de julgamentos, e direcionada a formação de vínculo se configura uma das ferramentas mais importante no tocante ao enfrentamento de práticas desumanizadas, muitas vezes precedidas de relação de poder historicamente produzidas que marcam o encontro entre profissionais e usuários do sistema de saúde.
Para que se realizem as considerações a despeito do objetivo geral, a saber: verificar o potencial da escuta qualificada como ferramenta de humanização do cuidado em saúde mental no âmbito da atenção básica em saúde no Brasil, é necessário levar em consideração os princípios e diretrizes da política nacional que direcionam o processo de humanização do Sistema Único de Saúde nos diversos níveis de atenção.

A PNH orienta-se pelos princípios da transversalidade, da indissociabilidade entre atenção e gestão, e também pelo princípio do protagonismo, corresponsabilidade e autonomia dos sujeitos e coletivos. Assim, deve estar inserida em todas as políticas e programas do SUS, e, por conseguinte, deve nortear as práticas de gestão e atenção básica em saúde mental, promovendo a valorização dos diversos sujeitos envolvidos no processo.

As diretrizes que conduzem os arranjos de trabalho sugeridos pela Política Nacional de Humanização são: o acolhimento; gestão participativa e cogestão; ambiência; clínica ampliada e compartilhada; valorização do trabalhador; e defesa do direito dos usuários. Para que tais diretrizes sejam exequíveis traduzindo-se em práticas humanizadas de saúde, a escuta qualificada precisa se configurar uma ferramenta técnica de todos os trabalhadores, das diversas especialidades, bem como, dos gestores.

No processo de acolhimento, por exemplo, para que se possa, de fato, estabelecer vínculo, compromisso multilateral e identificar as necessidades únicas da pessoa assistida, o diálogo precisa ser qualificado, o sujeito precisa ser escutado. No acolhimento efetivo, com a escuta adequada, é possível identificar que técnicas e tecnologias de fato vão suprir as demandas do usuário. A escuta qualificada torna a prática da clínica ampliada possível, pois de que outra maneira seria possível considerar a singularidade do sujeito e complexidade do processo saúde/doença, levando em consideração que o primeiro não se resume ao segundo? 


\section{APS em Revista}

Vol. 1, n. 2, p. 170/179 | Maio/Julho - 2019

ISSN 2596-3317 - DOI 10.14295/aps.v1i2.23

Santos, A. B.

Para tornar os cuidados em saúde mental humanizados, seja no nível primário, secundário ou terciário, é necessário escutar, mas escutar muito além do que as palavras são capazes de dizer, é necessário ouvir o que os gestos dizem, o que expressão grita, o que as marcas comunicam, é necessário escutar despindo-se de preconceitos e enxergando a pessoa assistida como um sujeito de direitos, e dentro das suas limitações, também, protagonistas da sua história, corresponsável, autônomo.

Em virtude dos fatos mencionados fica comprovada a hipótese de que a escuta qualificada constitui-se como uma ferramenta capaz de revolucionar a lógica tradicional do cuidado em saúde mental no âmbito da atenção básica, possibilitando a humanização das práticas de promoção e prevenção, diagnóstico, tratamento e reabilitação, ou seja, possibilitando que neste contexto que consideradas as circunstâncias sociais, éticas, educacionais e psíquicas dos sujeitos envolvidos.

\section{REFERÊNCIAS BIBLIOGRÁFICAS}

(1) FOUCALT, Michel. A história da Loucura. Tradutor: José Teixeira Coelho Netto. São Paulo: Editora Perspectiva, 1978

(2). GONÇALVES, Alda Martins; SENA, Roseni Rosângela de. A Reforma Psiquiátrica no Brasil: contextualização e reflexos sobre o cuidado com o doente mental na família. Rev Latino-am Enfermagem, [S. L.], v. 9, n. 2, p. 48-55, 2001. Disponível em <http://www.periodicos.usp.br/rlae/article/view/15 51/1596>. Acesso em: 10 set. 2018.

(3). FURTADO, Juarez Pereira; CAMPOS, Rosana Onocko. A transposição das políticas de saúde mental no Brasil para a prática nos novos serviços. Rev. Latinoam. Psicopat. Fund., [S. L.], v. 8, n. 1, p. 109-122, 2005. Disponível em <http://www.scielo.br/pdf/rlpf/v8n1/1415-4714rlpf-8-1-0109.pdf >. Acesso em: 12 set. 2018.
(4). FONSECA, Tania Mara Galli; JAEGER, Regina Longaray. Psiquiatrização da vida: Arranjos da loucura hoje. Rev. Pólis e Psique, Porto Alegre, v. 2 n. 3, p. 188-207, 2012. Disponível em: <https://seer.ufrgs.br/PolisePsique/article/view/40 327/25634>. Acesso em: 12 set. 2018.

(5). BRASIL. Ministério da Saúde. Secretaria de Atenção à Saúde. Departamento de Atenção Básica. Cadernos de Atenção Básica: Saúde mental. Brasília: Ministério da Saúde, 2013.

(6). BRASIL. Ministério da Saúde. Lei no. 10.216 de 06 de abril de 2001. Brasília: Diário Oficial da República Federativa do Brasil, 2001.

(7). BRASIL. Ministério da Saúde. Portaria no. 3.088, de 23 de dezembro de 2011. Brasília: Diário Oficial da República Federativa do Brasil, 2011.

(8). BRASIL. Ministério da Saúde. Portaria no. 2.436 de 21 de setembro de 2017. Brasília: Diário Oficial da República Federativa do Brasil, 2017.

(9). BRASIL. Ministério da Saúde. SecretariaExecutiva. Núcleo Técnico da Politica Nacional de Humanização. Humaniza SUS politica nacional de humanização. (versão preliminar). Brasília: Ministério da Saúde, 2001.

(10). FORTES, Paulo Antônio de Carvalho. Ética, direitos dos usuários e políticas de humanização da atenção à saúde. Saúde e Sociedade, [S.L.], v.13, n.3, p.30-35, 2004. Disponível em: <http://www.scielo.br/pdf/sausoc/v13n3/04.pdf>. Acesso em: 11 set. 2018.

(11). RIOS, Izabel Cristina. Humanização: a Essência da Ação Técnica e Ética nas Práticas de Saúde. Rev. Brasileira de Educação Médica, [S.L.], v. 33, n. 2, p. 253-261, 2008. Disponível em: <http://www.scielo.br/pdf/rbem/v33n2/13.pdf>. Acesso em: 10 set. 2018.

(12). SIMÕES, Ana Lúcia de Assis, et al. Humanização na saúde: enfoque na atenção primária. Texto Contexto Enferm., Florianópolis, v. 16, n. 3, p. 439-444, 2007. Disponível em: <http://www.scielo.br/scielo.php?script=sci_arttext \&pid=S0104-07072007000300009>. Acesso em: 20 set. 2018. 


\section{APS em Revista}

Vol. 1, n. 2, p. 170/179 | Maio/Julho - 2019

ISSN 2596-3317 - DOI 10.14295/aps.v1i2.23

Santos, A. B.

(13). BRASIL. Ministério da Saúde. SecretariaExecutiva Núcleo Técnico da Política Nacional de Humanização. Política Nacional de Humanização: Documento base para Gestores e Trabalhadores do SUS. Brasília, Ministério da Saúde, 2. ed, 2004.

(14). RAIMUNDO, Jader Sebastião; CADETE, Matilde Meire Miranda. Escuta qualificada e gestão social entre os profissionais de saúde. Rev. Acta Paul Enferm. v. 25, n. 2, p. 61-67, 2012. Disponível em: <http://www.scielo.br/pdf/ape/v25nspe2/pt_10.pd f >. Acesso em: 11 set. 2018.

(15). BRASIL. Ministério da Saúde. Secretaria de Atenção à Saúde. Política Nacional de Humanização PHN. Brasília: Ministério da Saúde, 1. ed., 2013b.

(16). SANTOS, Jussara da Paixão dos. A escuta qualificada - instrumento facilitador no acolhimento ao servidor readaptado. $24 \mathrm{f}$. Monografia (Especialização em Gestão de Pessoas). São Paulo: Programa FGV in company, 2014. Disponível em: <https://pt.scribd.com/document/373251596/TCCEscuta-Qualificada>. Acesso em 10 set. 2018.
(17). MAYNART, Willams Henrique da Costa. A escuta qualificada e 0 acolhimento na atenção psicossocial. Acta Paul Enferm, [S.L.], v.27, n. 4, p. 300-3, $2014 . \quad$ Disponível em: <http://www.scielo.br/pdf/ape/v27n4/1982-0194ape-027-004-0300.pdf>. Acesso em: 21 set. 2018.

(18). JORGE, Maria Salete Bessa, et al. Promoção da Saúde Mental - Tecnologias do Cuidado: vínculo, acolhimento, co-responsabilização e autonomia. Ciência \& Saúde Coletiva, [S. L.] v. 16, n. 7, p. 30513060, 2011. Disponível em: <http://www.scielo.br/pdf/csc/v16n7/05.pdf >. Acesso em: 15 set. 2018.

(19). MENDES, Isabel Amélia Costa, et al. Diagnóstico e prognóstico graves: dificuldades para comunicar ao paciente e à família. Cienc. Cuid. Saúde, [S.L.], v. 6, n. 3, p. 300-304, 2007. Disponível em: <http://periodicos.uem.br/ojs/index.php/CiencCuid Saude/article/view/3989/2720>. Acesso em: 15 set. 2018. 


\section{ABSTRACT}

Background: The guiding research hypothesis was that the bug qualified in primary care health is a tool able to revolutionize biomedical care logic.

Methods: This article guided by the precepts of a bibliographical research, in order to collect, analyze and interpret what has already been said, written or filmed about a qualified primary care listening and humanization in mental health. After the bibliographic survey she conducted an exploratory study with the aim of elucidating the phenomenon researched through the existing theoretical contributions.

Results: From this study, the guiding hypothesis, can be proven, and the specific objectives enabled the potential of skilled listening while humanizing care tool was explained in the course of the research.

Conclusions: To listen qualified allows the humanization of practices of promotion and prevention, diagnosis, treatment and rehabilitation in mental health, i.e. allows in this context that the social circumstances, ethical, psychological and educational of the subjects involved.

Keywords: Humanization. Mental Health. Basic Care. 